\title{
Course correction
}

\author{
The US Food and Drug Administration is sticking to its plan to carry out mandatory premarket review of all gene- \\ edited livestock, irrespective of trait risk. It should rethink.
}

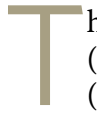
he US Food and Drug Administration (FDA) Center for Veterinary Medicine (CVM) continues to argue that every animal created by gene editing should be subject to mandatory premarket review and substantial safety testing. This position, first outlined in a 2017 draft guidance, presupposes that all gene-edited animal are potentially hazardous - despite the evidence and the views of many researchers. It canonizes a precautionary stance to gene-edited animals, irrespective of the trait engineered, even if the animal is indistinguishable from one created by conventional breeding. It is out of step with the practice of the US Department of Agriculture (USDA), which recently deferred from regulating gene-edited products. And it goes against decades of US policy focusing on appropriate product risks and benefits through the Coordinated Framework.

The FDA started its maverick journey to process-based, rather than product-based, oversight of genetically engineered animals in 2009, issuing a guidance under the new animal drug provisions of the Federal Food, Drug, and Cosmetic Act. In doing so, it departed from principles laid down by the Office of Science and Technology Policy in 1992 and before that in 1986. Then, in 2017, it doubled down, issuing draft guidance to regulate all gene-edited animals - with few exceptions - on the basis of the presence of any "intentionally altered genomic DNA."

In this issue, CVM Director Scott Solomon reiterates the agency's determination to regulate all gene-edited food animals. As support, he cites data published in this issue in which extraneous donor plasmid DNA is detected in one allele of Holstein dairy cattle edited at the POLLED locus to make them hornless (originally described in 2016). These data confirm observations in another paper published in our pages last year that offspring from these founder animals also contain the same insertion.

None of these data reveal anything about safety. As Solomon puts it: "the existence of an unintended alteration does not necessarily demonstrate that edits of an animal's genome are unsafe, either to the animal or to anyone consuming food from the animal." In over 30 years, there has yet to be a single compelling case where genetic engineering posed a safety problem in a food animal.

Solomon notes that bovine leukocyte adhesion deficiency (BLAD) syndrome - a lethal recessive disease that arose and was expanded by conventional breeding - is "illustrative of the risks of occult genomic alterations."

BLAD is not a justification for mandatory regulation of all gene-edited animals. If anything, it illustrates that conventional breeding might warrant tighter FDA oversight, especially when rapid breeding expansion programs thrust particular genetic profiles to the fore. So yes, the presence of extraneous donor plasmid in the gene-edited POLLED Holstein DNA was unexpected and initially missed. And gene editing is not the neat precise process that some proponents tout. But if the BLAD case history tells us anything, it is that the origin of a DNA arrangement (conventional breeding, recombinant DNA or gene editing) makes little difference to an animal. The genomes of domestic cattle contain millions of natural variants: the 1000 Bull Genomes Project found >86.5 million differences (insertions, deletions and single nucleotide variants) among cattle breeds. According to prominent researchers in the field, none of these variants has been shown to produce ill effects on consumers of milk or meat. Amidst this background of innocuous variation, how can the presence of one identifiable variant justify the costs and delays of mandatory FDA oversight?

Certainly, it makes no economic sense. A study from last year estimates that geneedited crops could take up to 14 years to reach the market and cost $\$ 24.5$ million; conventionally bred products get to market three times faster and cost half as much. Gene-edited animals will not be any different. The most valuable bull ever sold went last year for $\sim \$ 1.5$ million: that would be a negligible return for any group seeking to develop a gene-edited animal under FDA review that would form the basis for a breeding program.
So why, while speeding up biopharmaceutical approvals, is the FDA so cautious about genetically engineered animals?

One reason is there are very few companies in this sector to argue the case for genetically engineered (including geneedited) animals. Even the Biotechnology Innovation Organization's support for animal biotech has appeared to wane in recent years. On the other hand, there is a powerful and litigious anti-GMO/proorganic lobby that repeatedly challenges the legitimacy of regulatory rulings and attempts to block market access following approval. The world's first genetically engineered animal - AquaBounty's AquAdvantage salmon - was approved by the FDA five years ago. It still has not reached US consumers, despite recent court victories.

Life-saving biotech drugs gain wide support in Washington. But there is little political capital invested in backing geneedited animals. Food remains plentiful and, if anything, the public mood is shifting to vegetarianism and animal-substitute products. Scientists and breeders want to use new technology in agriculture, but public sentiment nostalgically harks back to Victorian farming practices in a way that it doesn't for Victorian medical practices.

A cautious, process-based regulatory route keeps the FDA out of trouble and lowers litigation risks for CVM's lawyers. But the agency could still alter course without reversing direction completely.

Mandatory oversight could be phased out to a system whereby the agency exercises discretion over which gene-edited animals are regulated according to the hazard represented by the introduced trait. This would be consistent with USDA policy and longstanding US regulatory policy. It would give the animal biotech sector a chance to bloom. And it would counter the narrative of fearmongers who would taint all geneedited animals as hazardous to public health and injurious to animal welfare.

Published online: 7 February 2020 https://doi.org/10.1038/s41587-020-0433-3 